\title{
Lineage-specific DNA methylation in T cells correlates with histone methylation and enhancer activity
}

\author{
Christian Schmidl, Maja Klug, Tina J. Boeld, Reinhard Andreesen, Petra Hoffmann, \\ Matthias Edinger, and Michael Rehli ${ }^{1}$ \\ Department of Hematology, University Hospital Regensburg, 93042 Regensburg, Germany
}

\begin{abstract}
DNA methylation participates in establishing and maintaining chromatin structures and regulates gene transcription during mammalian development and cellular differentiation. With few exceptions, research thus far has focused on gene promoters, and little is known about the extent, functional relevance, and regulation of cell type-specific DNA methylation at promoter-distal sites. Here, we present a comprehensive analysis of differential DNA methylation in human conventional $\mathrm{CD}^{+} \mathrm{T}$ cells (Tconv) and $\mathrm{CD} 4{ }^{+} \mathrm{CD} 25^{+}$regulatory $\mathrm{T}$ cells (Treg), cell types whose differentiation and function are known to be controlled by epigenetic mechanisms. Using a novel approach that is based on the separation of a genome into methylated and unmethylated fractions, we examined the extent of lineage-specific DNA methylation across whole gene loci. More than 100 differentially methylated regions (DMRs) were identified that are present mainly in cell type-specific genes (e.g., FOXP3, IL2RA, CTLA4, CD4OLG, and IFNG) and show differential patterns of histone $\mathrm{H} 3$ lysine 4 methylation. Interestingly, the majority of DMRs were located at promoter-distal sites, and many of these areas harbor DNA methylation-dependent enhancer activity in reporter gene assays. Thus, our study provides a comprehensive, locuswide analysis of lineage-specific methylation patterns in Treg and Tconv cells, links cell type-specific DNA methylation with histone methylation and regulatory function, and identifies a number of cell type-specific, CpG methylationsensitive enhancers in immunologically relevant genes.
\end{abstract}

[Supplemental material is available online at www.genome.org. The microarray data from this study have been submitted to Gene Expression Omnibus (GEO) (http://www.ncbi.nlm.nih.gov/geo/) under accession no. GSE14281.]

A cell's identity and its developmental potential are governed by epigenetic mechanisms that control chromatin structure and the accessibility of regulatory DNA sequences. Methylation of cytosine residues in genomic DNA is an important epigenetic mark that is essential for normal embryonic development in mammals (Okano et al. 1999), imprinting (Li et al. 1993), X-inactivation (Goto and Monk 1998), and silencing of potential hazardous genetic elements such as transposons (Walsh et al. 1998). In general, DNA methylation is linked to gene silencing, but its capacity to repress gene transcription depends on the surrounding sequence context and in particular on the local density of CpGs (Weber et al. 2007). The repressor function of CpG methylation is best studied for CpG-dense promoter regions (the so-called CpG islands) that are frequently silenced in cancer as a consequence of diseaseassociated aberrant CpG methylation (Plass and Soloway 2002; Herman and Baylin 2003). Its influence on gene expression in normal physiological settings is less well understood. The great majority of $\mathrm{CpG}$ islands are protected from CpG methylation in normal cells, probably due to the presence and function of general transcription factors such as specific protein (SP) 1 and 3 (Brandeis et al. 1994). There is substantial evidence that less CpG-dense promoter regions are more frequently targeted by DNA methylation and that gene expression and CpG methylation status often correlate (Schilling and Rehli 2007; Weber et al. 2007). Traditionally, the tissue- or cell type-specific DNA methylation studies focused on proximal promoter regions. However, a number of recent observations suggest that only a limited number of promoters display cell

\footnotetext{
${ }^{1}$ Corresponding author.

E-mail Michael.Rehli@klinik.uni-regensburg.de; fax 49-941-944-5593. Article published online before print. Article and publication date are at
} http://www.genome.org/cgi/doi/10.1101/gr.091470.109. type-specific CpG methylation, suggesting that DNA methylation might have a minor role in controlling cell type- or lineage-specific gene regulation (Meissner et al. 2008; Mohn et al. 2008).

With few exceptions, promoter-distal sequences have received little attention so far, and we know little about the global distribution and dynamics of DNA methylation during normal developmental processes, particularly in lineage specification and differentiation processes in adult organisms. To gain a better understanding of their biological role, CpG methylation patterns have to be studied globally in well defined model systems such as embryonic stem cells or the hematopoietic system. The latter is of particular interest for epigenetic studies, as progenitor cells as well as various differentiated cell lineages can be isolated and purified for a comparative analysis of homogenous cell subpopulations. With the development and therapeutic use of "epigenetic drugs" such as DNA methyltransferase or histone deacetylase inhibitors, it is of growing importance to understand the underlying regulatory mechanisms and potential effects on the normal hematopoietic cell system.

Natural $\mathrm{CD}^{+} \mathrm{CD} 25^{+}$regulatory $\mathrm{T}$ (Treg) cells play a fundamental role in maintaining immunological self tolerance and immune homeostasis (Vignali et al. 2008). They develop in the thymus as an independent $\mathrm{CD} 4^{+} \mathrm{T}$ cell lineage and represent a prime example for epigenetic regulation. It was shown that the functional program of Treg cells is at least partially controlled by miRNA pathways (Chong et al. 2008; Liston et al. 2008; Zhou et al. 2008), and continuous expression of the lineage-directing transcription factor FOXP3 is dependent on its DNA methylation status at a methylation-sensitive, Treg cell-specific enhancer (Floess et al. 2007; Kim and Leonard 2007; Polansky et al. 2008).

Apart from this particular region at the FOXP3 locus, we know little about the regulatory role of DNA methylation during 
Treg lineage commitment, differentiation, and cell type-specific gene regulation. Here, we describe a comprehensive comparative analysis of DNA methylation patterns at selected gene loci in human $\mathrm{CD} 4{ }^{+} \mathrm{CD} 25^{-}$conventional T cells (Tconv) and Treg cells. In line with recent observations in other cell systems (Meissner et al. 2008; Song et al. 2008), we found that the majority of differentially methylated regions (DMRs) are located at promoter-distal sites. Many of these areas were found to harbor DNA methylationdependent enhancer activity in reporter gene assays, and cell typespecific demethylation was found to correlate with increased methylation at histone $\mathrm{H} 3$ lysine 4 (H3K4).

Thus, we identified a number of cell type-specific, CpG methylation-sensitive enhancers at immunologically relevant genes in Treg and Tconv cells. In a more general point of view, our data suggest that the restriction of cell type-specific enhancers is a key function of DNA methylation in adult progenitor cells and that differentiation and lineage commitment are associated with specific methylation or demethylation events in such enhancer regions.

\section{Results}

Identification of differentially methylated regions (DMRs) in Treg and Tconv cells using methyl-CpG immunoprecipitation (MClp)

The recent development of fractionation techniques that enrich for methylated DNA fragments now permits the examination of CpG methylation on global platforms such as oligonucleotide tiling arrays or next-generation sequencers. Current technologies are particularly well suited to address CpG methylation in CpG-dense regions, whereas it remains difficult to systematically analyze the regions of lower CpG content that comprise the majority of mammalian genomes.

We previously developed technologies for the fractionation of genomic DNA fragments depending on their CpG density (MCIp; Gebhard et al. 2006; Schilling and Rehli 2007) and adapted this approach to identify regions that are differentially methylated in two closely related $\mathrm{T}$ cell populations, namely Treg and Tconv cells. Due to the low frequency of Treg cells in peripheral blood, we FACS-purified CD45RA ${ }^{+}$naïve $\mathrm{CD} 4^{+} \mathrm{CD} 25^{\text {high }}$ T cells and expanded those cells in vitro (see Methods). We previously showed that these cells homogeneously maintain all phenotypic, functional, and epigenetic Treg cell characteristics even after extensive in vitro proliferation (Hoffmann et al. 2006; Baron et al. 2007). We separated gDNA from both expanded Treg and Tconv cells $\left(\right.$ Treg $^{\exp }$ and Tconv ${ }^{\exp }$ ) into unmethylated (CpG) and methylated pools (mCpG) using MCIp and compared cell type-specific differences in DNA methylation by cohybridization of the two unmethylated or methylation]). the two methylated DNA subpopulations of Treg and Tconv cells, respectively, to locus-wide tiling arrays. As enriched DNA fragments from a cell type in the methylated fraction should be depleted in the unmethylated fraction, the signal intensities in CpG pool and mCpG pool hybridizations should complement each other ("mirror-image" approach; Fig. 1A) and thereby allow the identification of differentially methylated regions (DMRs). Because we expected to find lineage-specific methylation differences with greater probability in regions associated with differential transcriptional activity, we limited our analysis to gene loci that showed cell type-specific gene expression in Treg versus Tconv cells (both in vitro expanded or freshly isolated) plus several control regions that were equally expressed in both cell types. Gene loci were selected based on our own and previously published expression studies (Pfoertner et al. 2006; Hill et al. 2007) to mainly include those genes that are differentially expressed in freshly isolated (unstimulated) cells but also in ex vivo cultured and expanded T cell subsets that underwent several cycles of polyclonal TCR activation (Hoffmann et al. 2006). The microarray used in this study covered $12 \mathrm{Mb}$ of the human genome and contained 69 regions (with a median size of $100 \mathrm{~kb}$ ) and 128 proximal promoter regions and 181 genes, including a number of well known and functionally relevant genes such as CD4OLG, IFNG, FOXP3, $I L 2 R, C T L A 4$, etc. (the complete list of selected regions is given in Supplemental Table S1).
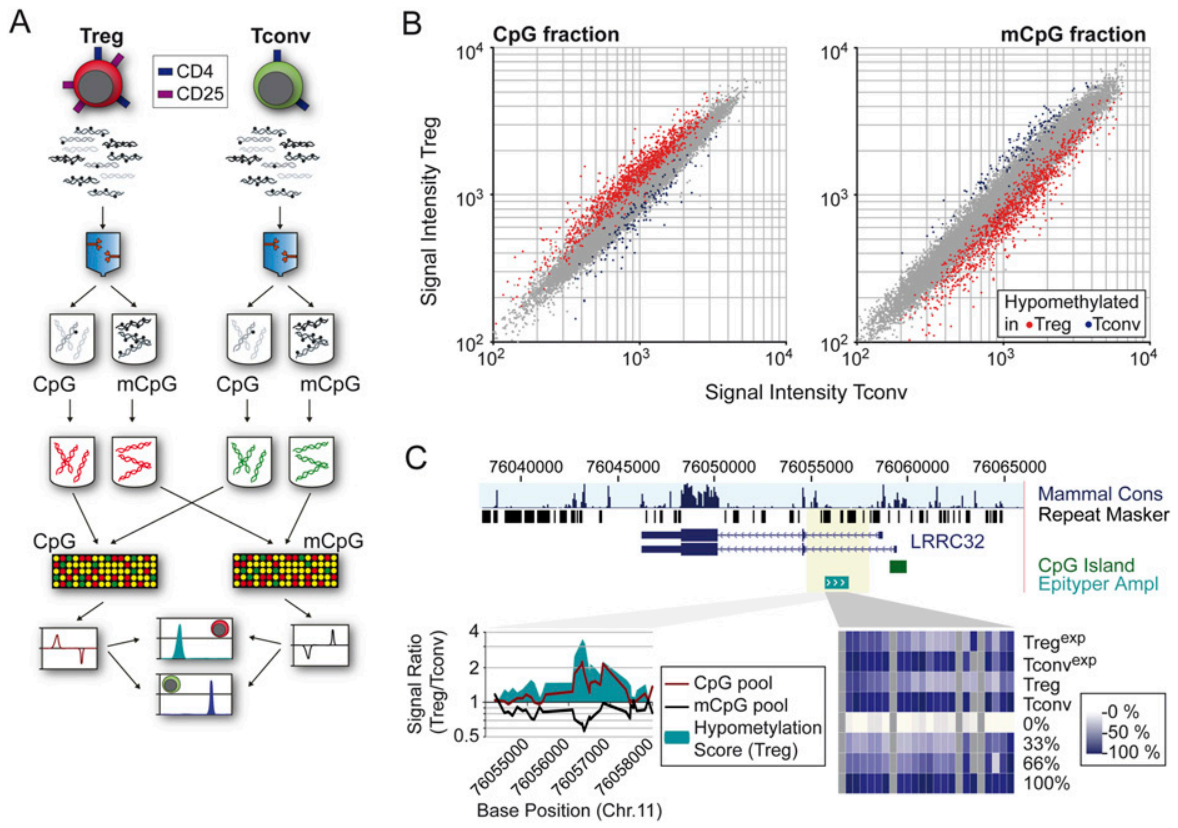

Figure 1. Locus-wide identification of DMRs using the MClp "mirror image" approach. $(A)$ Schematic outline of the MClp fragmentation and hybridization strategy. The fragmented genomes of Tconv and Treg cells are separated into unmethylated $(\mathrm{CpG})$ and methylated $(\mathrm{mCpG})$ pools. Each pool is directly labeled using fluorescent dyes, and each pool of one cell type is compared with the corresponding pool of the other cell type on a locus-wide microarray. Microarray images are compared to identify regions that show a reciprocal hybridization behavior. (B) Representative scatter plots of CpGand $\mathrm{mCpG}$-pool hybridizations are shown. Probes with reciprocal signal intensity ratios indicate the presence of DMRs. (Red) Treg cells; (blue) Tconv cells. (C) Exemplary validation of microarray results using mass spectrometry. The intron 1 region of $L R R C 32$ is enriched in the unmethylated (CpG, red line) and depleted in the methylated ( $\mathrm{mCpG}$, black line) pools of Treg cells. A large hypomethylation score (defined as the difference product of $\log _{10}$ signal intensity ratios of both hybridizations) indicates differential methylation (bottom left panel). The same region was analyzed by MALDI-TOF MS (Epityper), and results are shown as a heat map (the scale ranges from white [no methylation] to dark blue [100\%

\section{Genome Research} www.genome.org 
A representative scatter plot of comparative microarray hybridizations from unmethylated $(\mathrm{CpG})$ and methylated pools (mCpG) is shown in Figure $1 \mathrm{~B}$, where microarray probes showing the expected complementary behavior are colored in red (hypomethylated in Treg) and blue (hypomethylated in Tconv). In total, we identified 132 regions with lineage-specific CpG methylation that were associated with 53 genes (a complete list of DMRs is given in Supplemental Table S2). The majority of the DMRs (89\%) were of low CpG content (LCRs), and all residual sites are of intermediate $\mathrm{CpG}$ content using the classification described by Weber et al. (2007). Only seven out of 132 DMRs overlapped with known proximal gene promoters, $64 \%$ of all DMRs were located within gene bodies, and $36 \%$ were located in intergenic areas. As shown in Supplemental Figure S1, DMR status and differential mRNA expression status were significantly correlated regardless of the relative DMR position (intergenic/intragenic). Next, we searched for known sequence motifs enriched in T cell subsetspecific DMRs. Consensus sites enriched in DMRs as compared with the whole genome included cAMP-responsive ATF/CREB sites (in DMRs hypomethylated in Tconv cells) or STAT5 motifs (in DMRs hypomethylated specifically in Treg cells). Lists of the topranking motifs are provided in Supplemental Table S3.

To validate and quantify methylation differences, a representative set of DMRs was selected for MALDI-TOF MS analysis (for information on amplicons and MALDI-TOF MS results for all samples, see Supplemental Tables S4, S5). Mass spectrometry yields quantitative methylation data of short stretches of subsequent $\mathrm{CpG}$ in high throughput and consequently allows validation of large sample sets. An example of microarray and corresponding MS results is shown in Figure 1C. LRRC32, encoding the Treg cell-specific surface molecule GARP, which mediates suppressive function and FOXP3 induction (upon ectopic expression in naïve Tconv cells), contained a region in intron 1 that showed a hybridization pattern indicative of Treg cellspecific hypomethylation (Fig. 1C, bottom left panel): Microarray signal ratios of Treg-Tconv comparisons were high in the unmethylated pools (CpG) and low in the methylated pools (mCpG), resulting in a significant hypomethylation score. Mass spectrometry of bisulfite-treated DNA demonstrated that the center region was indeed completely methylated in Tconv cells and only weakly methylated in Treg cells, regardless of whether cells were freshly isolated or expanded (Fig. 1C, bottom right panel). In total, 26 out of 31 selected DMRs were confirmed by MS.

\section{Methylation levels at DMRs in other hematopoietic cell types}

To investigate whether the identified DMRs were specific for $T$ cell populations, we also obtained mass spectrometry data for other major blood cell types including $\mathrm{CD}^{+} \mathrm{T}$ cells, CD19+ $\mathrm{B}$ cells, $\mathrm{CD} 6^{+} \mathrm{NK}$ cells, CD $14^{+}$monocytes, and $\mathrm{CD} 4^{+}$hematopoietic progenitor cells, as well as the Jurkat T cell line. CD34 ${ }^{+}$cells represent a mixture of mainly committed myeloid, but also common hematopoietic progenitor, cells. As shown in Supplemental Figure S2, the majority of the CpG dinucleotides located in DMRs are methylated in $\mathrm{CD} 4^{+}$hematopoietic progenitor cells, suggesting that CpGs in T cell-specific DMRs are demethylated during progenitor cell differentiation. It is also noteworthy that the observed CpG methylation differences were detected both in freshly isolated T cell subsets as well as in T cells that were cultured and expanded in vitro. Since the latter involved a polyclonal TCR stimulation for both conventional and regulatory T cell subsets, it is likely that the observed differences are characteristic for each lineage and are not affected by T cell activation.

MS-derived hematopoietic methylation profiles are shown in detail for CTLA4 (Fig. 2), IL2RA (Fig. 3), and FOXP3, CD4OLG, $I F N G$, and LRRC32 (Supplemental Figs. S3-S6). When compared with other blood cell types, only a few regions were T cell typespecific, including two upstream regions of CTLA4 (Fig. 2), a region in intron 1 of IL2RA (Fig. 3), intron 1 of FOXP3 and intron 1 of the neighboring PPP1R3F (Supplemental Fig. S3), intron 4 of CD4OLG (Supplemental Fig. S4), and the downstream DMR of LRRC32 (Supplemental Fig. S6).

\section{Correlation between DNA methylation, chromatin boundaries, and histone methylation}

The majority of the detected DMRs were located at promoter-distal sites. To characterize the potential functions of these regions, we next examined the binding sites of the insulator protein CTCF. Binding of CTCF at imprinted loci restricts or directs enhancerpromotor interactions, and this binding is often regulated by DNA methylation (Bell and Felsenfeld 2000). We mapped CTCF binding sites using chromatin immunoprecipitation with microarray

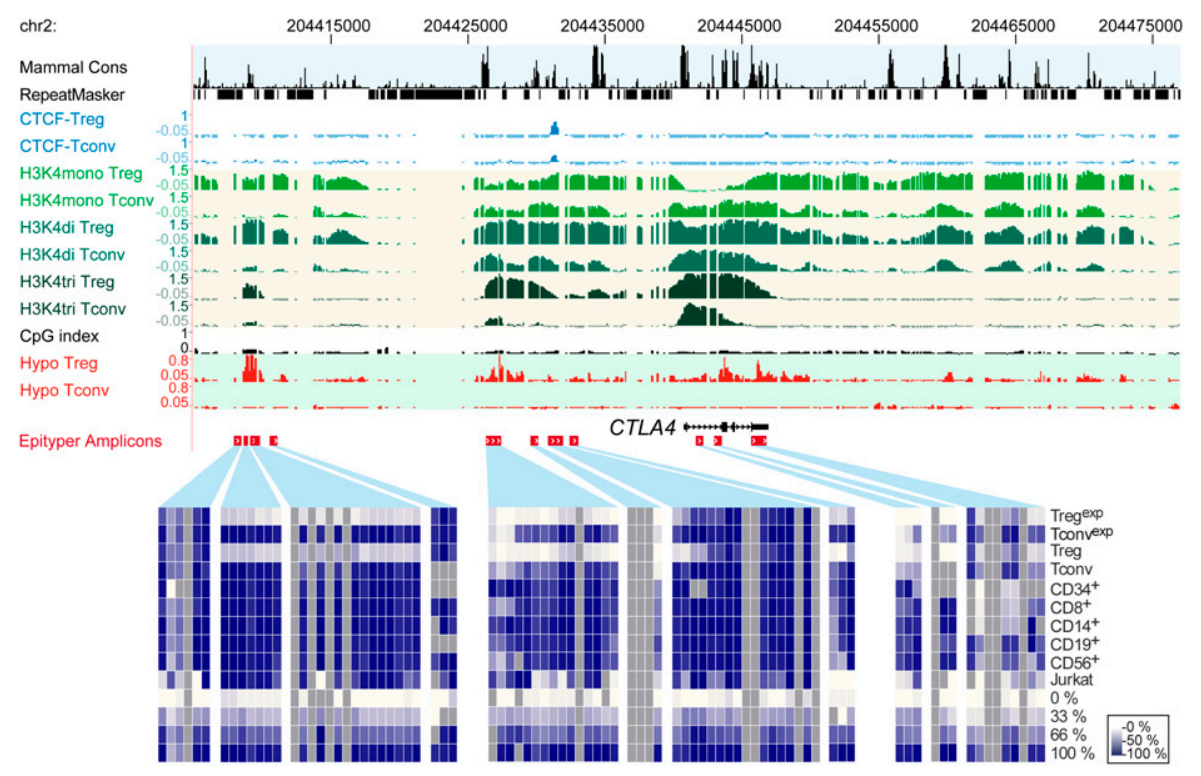

Figure 2. Chromatin modification and CTCF binding patterns across the CTLA4 gene locus. Shown are the following tracks (from top to bottom): mammalian Consensus (Cons, black); repetitive regions as identified by the RepeatMasker program (black); ChIP-chip tracks for CTCF (blue); monomethylated (pale green), dimethylated (green), and trimethylated (dark green) lysine 4 of histone $\mathrm{H} 3$; and the CpG index (indicating the methylation density $300 \mathrm{bp}$ up- or downstream of each microarray probe) as well as hypomethylation scores (red) for both cell types. Several amplicons were designed for MALDI-TOF MS analysis of bisulfite-treated DNA as indicated below the tracks. Methylation levels of individual CpGs in the indicated cell types are shown color-coded as described in the Fig. 1 legend. 


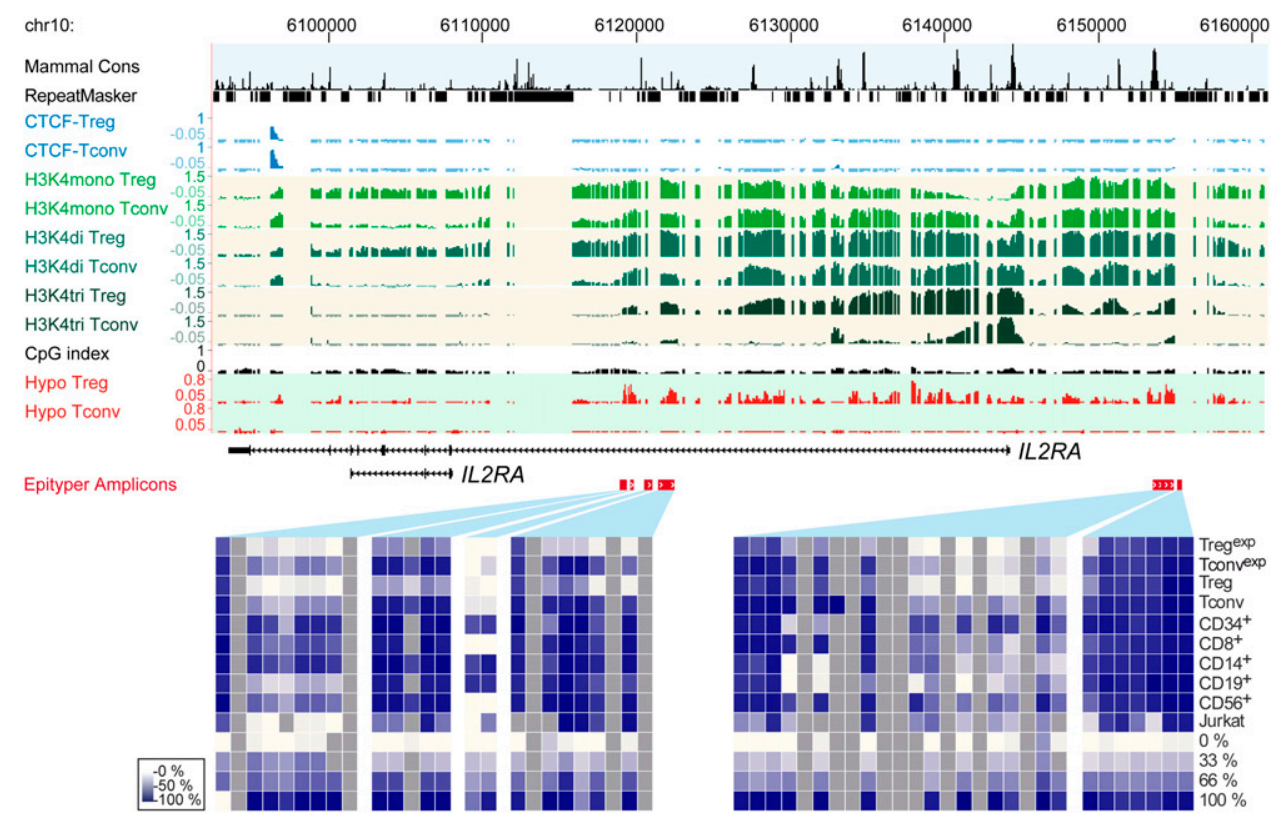

Figure 3. Chromatin modification and CTCF binding patterns across the IL2RA gene locus. Tracks and heat maps are shown as described in the legend of Fig. 2.

hybridization (ChIP-chip) on our locus-wide tiling array. The binding pattern of CTCF was almost identical to the published data set for $\mathrm{CD}^{+}{ }^{+} \mathrm{T}$ cells (Barski et al. 2007) and showed little variation between Treg and Tconv cells. In addition, we found no overlap between DMRs and CTCF binding (data not shown), suggesting that the regulation of CTCF-controlled chromatin boundaries is not a major function of DMRs at the nonimprinted loci investigated in this study.

To address the question of whether DMRs at promoter-distal sites harbor regulatory functions, we examined the association of methylation patterns with other chromatin modifications known to control enhancer elements. Mono- and dimethylation of histone $\mathrm{H} 3$ lysine 4 (H3K4) were previously shown to mark enhancer regions (Heintzman et al. 2007), whereas H3K4 trimethylation generally associates with transcription start sites (Barski et al. 2007). Using the ChIP-chip approach, we mapped these three histone marks in expanded Treg and Tconv cells. In general, we observed the expected continuous pattern of H3K4 methylation: Mono- and dimethylation were often found together and showed a similar distribution if no trimethylation was present. If trimethylation is detected, monomethylation tends to decrease (relative to dimethylation). Monomethylation without dimethylation, or trimethylation without dimethylation, were rarely detected. Examples of selected gene loci are presented in Figures 2 and 3, as well as in Supplemental Figures S3-S6 (the complete set of microarray data is provided as UCSC Genome browser track files in the Supplemental material).

Since the three possible methylation states of H3K4 are not independent from each other, they cannot be correlated with differential CpG methylation independently. However, if DMRs correlated with H3K4 methylation, one would expect to observe a co-enrichment of cell type-specific hypomethylation and H3K4 methylation in isolates from the same cell type. The diagrams in Figure 4 show that this is indeed the case. Di- and trimethylated H3K4 clearly correlated with the differential methylation status at sites where H3K4 methylation was observed. Due to the in- terdependence of $\mathrm{H} 3 \mathrm{~K} 4$ methylation states and the frequent appearance of di- and trimethylation states, monomethylated H3K4 did not correlate with the differential methylation status. We also classified DMR regions according to their relative H3K4 methylation status. Pie charts in Figure 4D illustrate the distribution of H3K4 methylation patterns at cell type-specific DMRs. Regions with activating regulatory function are most likely in those classes where hypomethylation in one cell type correlates with an increased level of H3K4 methylation in the same cell type. The distribution of H3K4 methylation patterns at cell type-specific DMRs depending on their relative position (intergenic/intragenic) are shown in Supplemental Figure S7. In line with previous observed distribution of global patterns (Barski et al. 2007; Heintzman et al. 2007), trimethylation of H3K4 was more strongly associated with intragenic DMRs.

\section{Enhancer activity of T cell-specific DMRs}

As methylated H3K4 has previously been associated with enhancer activity (Heintzman et al. 2007) and co-segregated with DMRs in our experiments, we next asked whether DMRs associate with enhancer activity. Properties of generic enhancers include their ability to increase transcriptional activity in a heterologous context, which can be studied using traditional reporter gene assays. We recently developed a reporter vector that completely lacks CpG dinucleotides (Klug and Rehli 2006) and utilized this system to test for heterologous enhancer activity of 24 selected DMRs. We preferentially selected DMRs that were associated with genes that also showed differential gene expression and H3K4 methylation (general properties of the selected DMRs are listed in Supplemental Table S7). Transient transfections were performed in untreated, PMA/ionomycin-treated, or PHA-treated Jurkat T cells using unmethylated (CpG) or in vitro SssI methylated (mCpG) reporter plasmids. As shown in Figure 5, 12 out of 24 DMRs significantly enhanced the activity of the basal (CpG-free) EF1 promoter. Importantly, all regions lost enhancer activity when 
A

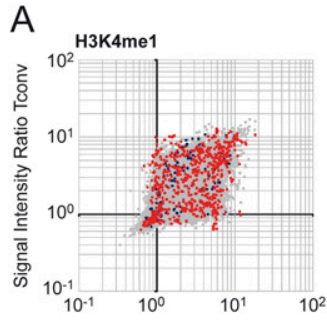

B
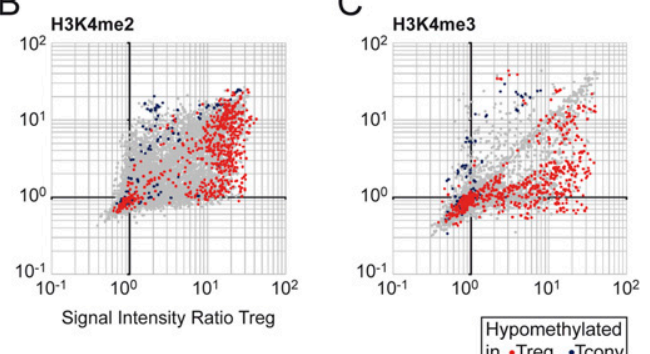

D

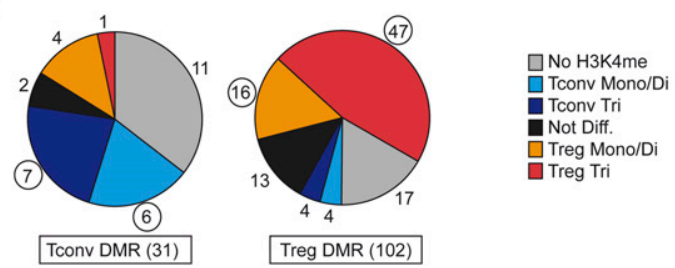

Figure 4. Correlation of DNA demethylation and $\mathrm{H} 3 \mathrm{~K} 4$ methylation status. $(A-C)$ Probe signal ratios of Tconv and Treg cells are plotted against each other for ChIP-chip experiments of monomethylated $(A)$, dimethylated $(B)$, and trimethylated $(C)$ lysine 4 of histone $\mathrm{H} 3$. Probes that appear along the diagonal indicate similar $\mathrm{H} 3 \mathrm{~K} 4$ methylation levels, whereas probes above or below the diagonal indicate higher methylation levels in Tconv or Treg cells, respectively. Probes in DMRs: (blue) unmethylated in Tconv; (red) unmethylated in Treg; (gray) all other probes. (D) The two pie charts illustrate the relationship of associated H3K4 methylation and DMRs hypomethylated in Tconv (left) or Treg (right). The H3K4 methylation status was classified as follows: DMRs with increased H3K4 trimethylation in Treg or Tconv cells (Treg Tri or Tconv Tri, respectively); DMRs with increased H3K4 mono- or dimethylation, but no trimethylation, in Treg or Tconv cells (Treg or Tconv Mono/Di respectively); DMRs with H3K4 methylation present but no difference between T cell subsets (Not diff.); and DMRs with no detectable H3K4 methylation (No H3K4me). The numbers of DMRs in each subclass are shown next to each piece of pie. Circled numbers indicate subclasses where hypomethylation in one cell type correlates with an increased level of H3K4 methylation in the same cell type.

methylated, suggesting that their activity is critically dependent on their CpG methylation status. Functionality in the enhancer assay did not correlate with DMR positioning (intergenic/intragenic), conservation status, the presence of DNase I hypersensitive sites in CD4+ T cells (Boyle et al. 2008), or H3K4 methylation status (for details, see Supplemental Table S7). However, the majority of regions that did not show enhancer activity in Jurkat cells corresponded to Treg cell-specific DMRs, including, e.g., both upstream CTLA4 regions and an upstream region of IKZF2 (also known as ZNFN1A2). In line with this, DMRs that did show enhancer activity in Jurkat cells were enriched for Tconv cell-associated consensus binding sites (cAMP-responsive ATF/CREB sites and ELK1 sites; for a list of the top ranking motifs, see Supplemental Table S8). Since Jurkat T cells represent a leukemic counterpart of conventional $\mathrm{T}$ cells, it is possible that they lack Treg cell-specific transcription factors that are necessary for enhancer functions of these regions. However, some Treg cell-specific DMRs did function even in Jurkat cells, suggesting that the relevant transcription factors required for enhancer activity at these sites were available.

\section{Discussion}

In this study we utilized a combination of DNA methylationdependent genome fractionation using MCIp and quantitative methylation analysis on a mass spectrometry platform (Sequenom MassARRAY system) to identify differentially methylated regions (DMRs) in two closely related $\mathrm{T}$ cell subtypes (Treg and Tconv cells). We identified more than 100 DMRs in 69 selected gene loci neglected in previous studies. Our findings in $\mathrm{CD} 4^{+} \mathrm{T}$ cells are in line with a recent study in ES cells that also identified promoterdistal regions as the main sites of dynamic changes in DNA methylation upon differentiation (Meissner et al. 2008), and with a study on tissue-specific CpG island methylation demonstrating that methylation of CpG islands in normal tissues preferentially occurs at promoter-distal sites (Illingworth et al. 2008). Most DMRs that we identified were of low CpG content (LCR), suggesting that promoter-distal elements may differ from promoter-proximal sites, where cell type-specific changes more frequently occur in intermediate $\mathrm{CpG}$ content regions (Mohn et al. 2008). Interestingly, DMRs were significantly enriched for transcription factor binding motifs that were previously shown to play a role in each $\mathrm{T}$ cell subset. DMRs hypomethylated in Tconv cells were enriched for ATF/CREB consensus sites, which are known to mediate mitogenic and CD28-dependent signals (Hsueh et al. 1997). Regions specifically demethylated in Treg cells were enriched for STAT5 consensus sites. Treg cell survival critically depends on the presence of IL-2. The transcription factor STAT5 is activated through the IL-2 receptor (Hou et al. 1995), has an essential role in $\mathrm{CD} 25^{+} \mathrm{CD} 4^{+}$regulatory T cell homeostasis (Antov et al. 2003), and is known to regulate the lineage-specific transcription factor FOXP3 through an intronic, methylation-sensitive enhancer (Zorn et al. 2006). The significant enrichment of consensus sites does not necessarily imply a biological significance. Further experiments are needed to show that these motifs are actually bound by transcription factors in vivo. However, the fact that enriched consensus motifs belong to transcription factors with known importance in each lineage may point to a functional role of lineage-specific DMRs. 
A

A

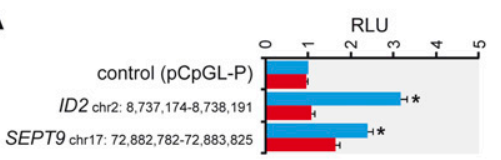

B

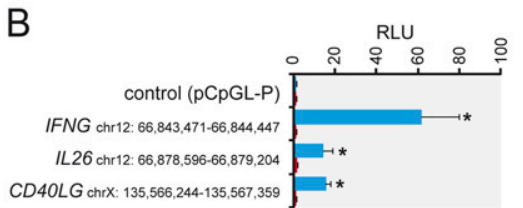

C

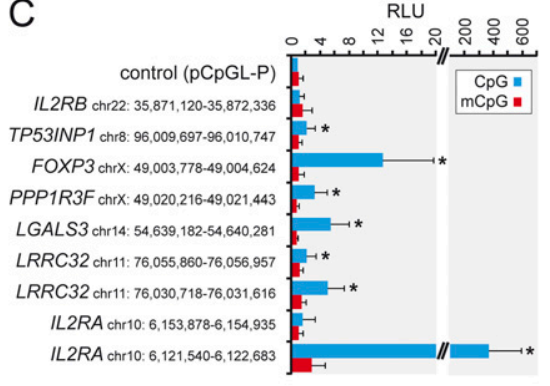

Figure 5. $\mathrm{CpG}$ methylation-dependent enhancer activity of selected DMRs. Several DMRs were cloned upstream of a basic EF1-promoter into the CpG-free luciferase vector PCpGL-P. The indicated plasmids were in vitro Sssl-methylated $(\mathrm{mCpG})$ or unmethylated $(\mathrm{CpG})$ and transiently transfected into lurkat T cells that were left untreated $(A)$, or were stimulated with PMA and ionomycin $(B)$ or PHA $(C)$ after transfection. Luciferase activity was normalized against the activity of a cotransfected Renilla construct, and mean values \pm SD are shown relative to the unmethylated pCpGL-P. $\left({ }^{*}\right)$ Significant difference between methylated and unmethylated plasmids $(P<0.05$, paired Student's $t$-test).

The majority of DMRs in both T cell subsets were methylated in $\mathrm{CD}_{3} 4^{+}$hematopoietic progenitor cells and often also methylated in other mature hematopoietic lineages. $\mathrm{CD}_{3} 4^{+}$cells comprise a relatively heterogeneous mixture of committed and uncommitted progenitor cells. However, the percentage of uncommitted as well as T cell lineage-committed precursors is likely to be small. Since we were unable to obtain sufficient numbers of primary human T cell progenitors, it is unclear whether individual methylation patterns are created by methylation of previously unmethylated $\mathrm{CpG}$ residues or by demethylation of previously methylated sites. Because CpG methylation is often found in $\mathrm{CD} 34^{+}$progenitors and mature cells of both myeloid and lymphoid lineages, it is likely that, in many cases, methylation marks are removed during lineage commitment of either T cell subtype.

Previous studies suggested that mono-, di-, or trimethylation at lysine 4 of histone H3 (H3K4) at promoter-distal sites is often associated with enhancer function (Barski et al. 2007; Heintzman et al. 2007; Wang et al. 2008b). In line with the findings of a recent study (Meissner et al. 2008), we observed an enrichment of H3K4 methylation (both di- and trimethylation) at many DMRs in the T cell type that was hypomethylated, which suggested a regulatory function for these regions. DMRs most likely will be associated with an active regulatory function when hypomethylation in one cell type correlates with an increased level of H3K4 methylation in the same cell type. However, since some gene regulatory events during the differentiation of conventional and regulatory T cells may be only of transient nature, it is possible that DMRs displaying no H3K4 methylation in our analysis have lost this dynamic histone mark but retained the more stable CpG methylation pattern. Converse patterns (hypomethylation in one cell type correlates with an increased level of H3K4 methylation in the other cell type) are rarely observed but are validated in at least one case: the DMR located upstream of ID2 (chr2:8735102-8735444) shows hypomethylation in Treg cells but increased H3K4 methylation in Tconv cells. The biological significance of the converse patterns is unclear; however, it is possible that silencing elements may also be subject to regulation by DNA methylation.

Three of the identified DMRs were previously described as functional enhancer elements. Both upstream regions at the IFNG/IL26 locus were defined as conserved, activation-induced enhancers in conventional murine $\mathrm{CD}^{+} \mathrm{T}$ cells (Schoenborn et al. 2007). In Treg cells, a functionally important intronic enhancer of the FOXP3 gene was shown to be methylation-sensitive
(Baron et al. 2007; Floess et al. 2007; Kim and Leonard 2007). Therefore, we now asked whether other DMRs might also demonstrate enhancer properties and whether methylation would restrict enhancer activity. Half of the 24 tested DMRs significantly enhanced the activity of a heterologous promoter in transient reporter gene assays performed in a T cell leukemia line (Jurkat). Most strikingly, all regions lost enhancer activity upon CpG methylation. Even some Treg-specific DMRs showed enhancer activity in the Jurkat cells, suggesting that these cells per se express the required factors for enhancer function and that CpG methylation may be critical to restrict the lineage-specific enhancer function of these DMRs in Tconv cells.

In some cases, DMRs (e.g., both DMRs upstream of CTLA4) co-located with trimethylated H3K4 that was not associated with detectable transcription (no associated ESTs or CAGE tags published in human or mouse; data not shown). Trimethylated H3K4 is usually associated with promoters, and its occurrence at enhancers is debated. Whereas Heintzman et al. (2007) preferentially found $\mathrm{H} 3 \mathrm{~K} 4$ monomethylation (and little or no H3K4 trimethylation) at p300-associated enhancers, Barski et al. (2007) identified all three methylation states at functional enhancers. It is therefore unclear whether the promoter-distal, H3K4 trimethylated sites identified in this study associate with so far uncharacterized functional transcription units, or whether they act as (transcribed) enhancer regions such as, e.g., the upstream enhancer of the myeloid- and B cell-specific SPI1 gene (Hoogenkamp et al. 2007). Since Treg cells represent only a minor fraction of $\mathrm{CD} 4^{+} \mathrm{T}$ cells, their transcriptome is likely underrepresented in public cDNA sequence databases. Therefore, further studies aiming at understanding cell type-specific gene regulation in T cell subtypes will require a more comprehensive definition of transcription units, especially in Treg cells.

A recent comprehensive study of the regulatory potential of mammalian conserved noncoding sequences suggests that only a small proportion of these regions can be expected to exhibit classical cis-regulatory activity in standard experimental assays (Attanasio et al. 2008). In our hands, only a minority of the identified DMRs were conserved during evolution. For example, the intronic DMR of IL2RA was not conserved across species but acted as the strongest enhancer in heterologous reporter assays. Our data suggest that conservation-centered approaches to identify enhancer elements may miss out on a large number of important regulatory sites.

In addition to the basic findings on cell type-specific DNA methylation described above, our study identifies a number of putative regulatory elements in genes that are highly important for $\mathrm{T}$ cell function. For example, we found a methylationsensitive enhancer in intron 4 of CD $40 L G$ in Tconv cells. The encoded cell surface receptor plays an important role in regulating B cell function through its interaction with CD40 on B cells and dendritic cells (vanKooten and Banchereau 1997). The majority of interesting regions, however, were associated with genes important for Treg cell biology. It was previously shown that sustained expression of the lineage-determining transcription factor FOXP3 is dependent on its DNA methylation status at 
a methylation-sensitive, Treg cell-specific enhancer in intron I (Baron et al. 2007; Floess et al. 2007; Kim and Leonard 2007). Our locus-wide analysis identifies an extensively demethylated area at this locus that extends into the neighboring protein phosphatase 1 , regulatory (inhibitor) subunit $3 \mathrm{~F}(P P P 1 R 3 F)$ gene, where we identified an additional methylation-dependent enhancer. Two DMRs with enhancer function were identified at the $L R R C 32$ locus encoding a recently described surface molecule (also called GARP) that seems to contribute to the suppressive function of Treg cells (Wang et al. 2008a). A novel and potent enhancer was found at the IL2RA gene, which encodes the alpha chain of the IL-2 receptor (CD25). This methylation-sensitive enhancer region was specifically demethylated in both freshly isolated Treg cells and Treg cells that were cultured and expanded in vitro. Since cultured and expanded conventional T cells express high levels of CD25 as a consequence of TCR activation, this region may contribute to regulating constitutive (rather than activation-induced) CD25 expression in Treg cells.

Additional DMRs were found upstream of $I K Z F 2$, encoding a regulator of lymphocyte development (Dovat et al. 2005), and CTLA4, encoding a molecule that is constitutively expressed on Treg cells and that suppresses immune responses by affecting the activating potency of antigen-presenting cells (Wing et al. 2008). Although both upstream CTLA4 regions and the upstream region of IKZF2 showed no enhancer activity in transient transfection assays, the exclusive DNA demethylation and the increased H3K4 methylation in Treg cells indicate a functional importance of these regions. Their activation may actually require Treg-specific trans-acting factors that are not present in the Jurkat cells used for the reporter gene studies. Further characterization of the identified DMRs, including the identification of DNA-binding factors mediating the observed enhancer activities, will likely reveal important insights into cell type-specific gene regulation in $\mathrm{T}$ cells.

In conclusion, the observed distribution of DMRs at promoterdistal regions, their association with functional chromatin marks, and, most strikingly, their methylation-sensitive enhancer activity suggest a role for DNA methylation in controlling lineage-specific gene expression, mainly by restricting promoter-distal regulatory elements. This basic principle is likely not confined to the two closely related $\mathrm{T}$ cell populations, but may apply generally to somatic cell lineages in adult organisms.

\section{Methods}

\section{Cell purification and culture}

Mononuclear cells (MNCs) were isolated from leukapheresis products of healthy volunteers (after their informed consent and in accordance with protocols approved by the local authorities) by density gradient centrifugation over Ficoll/Hypaque (Biochrom $\mathrm{AG})$. $\mathrm{CD} 4^{+}$cells were enriched using magnetically labeled human CD4 MicroBeads (Miltenyi Biotec) and the Midi-MACS system (Miltenyi Biotec). The $\mathrm{CD}^{+}$fraction was stained with CD4-FITC (SK3), CD25-PE (2A3), and CD3-APC (UCHT1) and separated into $\mathrm{CD}^{+} \mathrm{CD} 4^{+} \mathrm{CD} 25^{-}$conventional $\mathrm{T}$ cells and $\mathrm{CD} 3^{+} \mathrm{CD} 4^{+} \mathrm{CD} 25^{\text {high }}$ regulatory $\mathrm{T}$ cells on a FACS-Aria high-speed cell sorter (BD Biosciences). The CD4- fraction was stained with CD19-FITC (4G7), CD56-PE (B159), CD3-PerCP (SK7), and CD8-APC (SK1) and sorted into $\mathrm{CD} 19^{+} \mathrm{CD}^{-} \mathrm{B}$ cells, $\mathrm{CD} 56^{+} \mathrm{CD}^{-}{ }^{-} \mathrm{NK}$ cells, and $\mathrm{CD}^{+}{ }^{+} \mathrm{CD} 8^{+} \mathrm{T}$ cells. Monocytes were enriched from MNC using counter-current elutriation in a J6M-E Beckman centrifuge (Beckman) with a large chamber and a JE-5 rotor at $1100 \mathrm{~g}$ at a flow rate of $110 \mathrm{~mL} / \mathrm{min}$ in
Hanks' balanced salt solution as described previously. Enriched cell fractions were stained with CD14-PE (M P9) and CD3-APC (UCHT1), and $\mathrm{CD} 14^{+} \mathrm{CD}^{-}$monocytes were further purified by FACS. All antibodies used were from BD Biosciences. All staining was performed in PBS/2\% FCS. Dead cells were excluded by staining with propidium iodide. All cell populations showed a purity of $>95 \%$ upon reanalysis.

$\mathrm{T}$ cell populations for expansion cultures were isolated as described in detail before (Hoffmann et al. 2006). In brief, peripheral blood mononuclear cells (PCMCs) were stained with anti$\mathrm{CD} 25-\mathrm{PE}$, and $\mathrm{CD} 25^{+}$cells were enriched by the use of anti-PE magnetic beads and the Midi-MACS system (Miltenyi Biotec). $\mathrm{CD} 4^{+} \mathrm{CD} 25^{-}$Tconv cells were FACS-purified from the CD25depleted cell fraction after staining with anti-CD4-FITC, and $\mathrm{CD} 4{ }^{+} \mathrm{CD} 25^{\text {high }} \mathrm{CD} 45 \mathrm{RA}^{+}$Treg cells were sorted from the CD25enriched population after staining with anti-CD4-FITC and antiCD45RA-APC. Reanalysis after sorting showed a purity of $>98 \%$.

FACS-purified Tconv and Treg cell populations were polyclonally expanded in vitro for $11-14 \mathrm{~d}$ as previously described (Hoffmann et al. 2004). Briefly, cells were stimulated with antiCD3 (OKT3; a kind gift from Janssen-Cilag) and anti-CD28 (CD28.2; BD Biosciences) antibodies presented by CD32-expressing L 929 cells in the presence of high-dose recombinant human IL-2 (rhIL-2, $300 \mathrm{U} / \mathrm{mL}$; Proleukin, Chiron). To exclude any contamination by feeder cells, all cultured populations were stained with CD4-FITC and PI and were FACS-sorted immediately prior to DNA isolation.

Jurkat cells (human T cell leukemia) were grown in 90\% 1640 RPMI (PAN Biotech GmbH) plus 10\% fetal bovine serum (FBS) supplemented with $2 \mathrm{mM}$ L-glutamine (Biochrome), MEM nonessential amino acids (GIBCO), sodium pyruvate (GIBCO), MEM vitamins (GIBCO), $50 \mathrm{U} / \mathrm{mL}$ penicillin/streptomycin (GIBCO), and $50 \mathrm{nM} 2$-mercaptoethanol (GIBCO) in a humified incubator at $37^{\circ} \mathrm{C}$ and $5 \% \mathrm{CO}_{2}$.

\section{RNA and DNA preparation}

Total cellular RNA of the different cell types was isolated using the RNeasy Kit (Qiagen). RNA concentration was measured with a ND1000 Spectrophotometer (NanoDrop, Thermo Fisher Scientific) and quality was controlled on agarose gels or using the Bioanalyzer (Agilent). Genomic DNA was prepared using the Qiagen Blood \& Cell Culture DNA Kit or the Qiagen DNeasy Blood \& Tissue Kit when working with smaller cell numbers. DNA concentration was determined with the NanoDrop spectrophotometer and quality was assessed by agarose gel electrophoresis.

\section{T cell transcriptome analysis}

RNA preparations from cultures of Treg and Tconv cells from four independent donors, as well as RNA preparations of freshly sorted Treg and Tconv cells from three independent donors, were analyzed using Whole Human Genome Oligo Microarrays (Agilent). Labeling and hybridization were performed using the Agilent Gene Expression system according to the manufacturer's instructions. In brief, $200-1000$ ng of high-quality RNA was amplified and Cyanine 3-CTP labeled with the One Color Low RNA Input Linear Amplification Kit (Agilent). Labeling efficiency was controlled using a NanoDrop spectrophotometer, and $1.65 \mu \mathrm{g}$ of labeled cRNA was fragmented and hybridized on the Whole Human Genome Expression Array (4x44K, Agilent). Images were scanned immediately after washing using a DNA microarray scanner (Agilent), processed using Feature Extraction Software 9.5.1 (Agilent), and further analyzed using GeneSpring GX software. Microarray data have been submitted and are available from the NCBI/GEO 
repository (accession no. GSE14281). Median normalized expression ratios for genes associated with DMR are given in Supplemental Table S2. A detailed description of the T cell transcriptome analysis will be published elsewhere.

\section{Methyl-CpG immunoprecipitation (MCIp)}

The recombinant MBD-Fc protein was produced as previously described (Gebhard et al. 2006). MCIp was performed as described with slight modifications (Schilling and Rehli 2007). In brief, genomic DNA was sonicated to a mean fragment size of 350-400 bp. Four micrograms of each sample was incubated with $200 \mu \mathrm{L}$ of Protein A-Sepharose 4 Fast Flow beads (GE Healthcare) coated with $80 \mu \mathrm{g}$ of purified MBD-Fc protein in $2 \mathrm{~mL}$ of Ultrafree-MC centrifugal filter devices (Amicon/Millipore) for $3 \mathrm{~h}$ at $4^{\circ} \mathrm{C}$ in buffer containing $300 \mathrm{mM} \mathrm{NaCl}$. Beads were centrifuged to recover unbound DNA fragments (300 $\mathrm{mM}$ fraction) and subsequently washed with buffers containing increasing $\mathrm{NaCl}$ concentrations (350, 400, 450, and $1000 \mathrm{mM})$. All fractions were desalted using the MinElute PCR purification kit (Qiagen). The distribution of CpG methylation densities of individual MCIp fractions was controlled by qPCR using primers covering the imprinted SNRPN and a genomic region lacking CpGs (empty6.2). Fractions containing unmethylated DNA (300-400 $\mathrm{mM}$ ) or methylated DNA $(\geq 450 \mathrm{mM}$ ) were pooled before subsequent labeling.

\section{Chromatin immunoprecipitation (ChIP)}

ChIP analysis of expanded and sorted Treg and Tconv cells was performed essentially as described (Metivier et al. 2003). Precipitation of pre-cleared chromatin from $2 \times 10^{6}$ cells was done overnight at $4^{\circ} \mathrm{C}$ using $2 \mu \mathrm{g}$ of anti-histone $\mathrm{H} 3$ lysine 4 monomethyl (Abcam), anti-histone H3 lysine 4 dimethyl (Upstate), anti-histone H3 lysine 4 trimethyl (Upstate), anti-CTCF (a gift from V. Lobanenkow, NIAID, NIH, Rockville, MD), and anti-rabbit IgG (Upstate). After reversion of crosslinks, enriched DNA fragments were recovered using the Qiaquick PCR-purification kit (Qiagen). The quality of each ChIP was controlled at known target sites by qPCR. For ChIP-chip analysis, all samples as well as an aliquot of equally treated input DNA were amplified by ligationmediated PCR (LM-PCR) for subsequent labeling.

\section{Ligation-mediated PCR (LM-PCR)}

ChIP and input DNA were blunted and phosphorylated for $30 \mathrm{~min}$ at $20^{\circ} \mathrm{C}$ in $50-\mu \mathrm{L}$ reactions containing $10 \times \mathrm{T} 4$ DNA ligase buffer, $2 \mu \mathrm{L}$ of dNTP mix (10 mM each), $3 \mathrm{U}$ of T4 DNA polymerase, $10 \mathrm{U}$ of $\mathrm{T} 4$ polynucleotide kinase, and $1 \mathrm{U}$ of Klenow DNA polymerase. Purification using the Qiaquick PCR purification kit (Qiagen) was followed by the addition of adenine to $3^{\prime}$-ends using Klenow fragment ( $3^{\prime}$ to $5^{\prime}$ exo minus) and dATP (1 mM) for $30 \mathrm{~min}$ at $37^{\circ} \mathrm{C}$. After clean-up using the MinElute kit (Qiagen), DNA fragments were ligated to linker DNA $(60 \mu \mathrm{M}$ pre-annealed JW102s 5'-GCGGTGACCCGGGAGATCTGAATTCT-3' and JW103 5'-GAATTCAGATC-3') with $4 \mu \mathrm{L}$ of DNA Quick-Ligase (NEB) in a $30-\mu \mathrm{L}$ reaction for $15 \mathrm{~min}$ at room temperature. Samples were cleaned up (Qiaquick PCR purification kit, Qiagen) and amplified using Phusion polymerase and JW102s oligonucleotide for 15 cycles. The product of the first amplification was diluted 1/20 with ddH2O, and $5 \mu \mathrm{L}$ of the dilution was used for a second round of amplification (15 cycles) using Phusion polymerase HOT START and JW102s oligonucleotide. The amplified ChIP and input material was purified using a Qiaquick PCR purification kit (Qiagen).

\section{Design, handling, and analysis of locus-wide microarrays}

Fifty-one highly regulated genes and 18 control genes were selected based on mRNA expression profiles of freshly sorted and expanded $\mathrm{T}$ cell subsets. Custom tiling arrays were designed for the 69 selected loci using the eArray web tool (https://earray. chem.agilent.com/earray/). Fluorescently labeled DNA for microarray hybridization of DNA pools from MCIp or LM-PCR amplified ChIP samples $(1 \mu \mathrm{g}$ of amplified DNA) was generated by direct labeling with Alexa Fluor 555-aha-dCTP and Alexa Fluor 647-aha-dCTP using the BioPrime Plus Array CGH Genomic Labeling System (Invitrogen). Hybridization and washing was performed as recommended by the manufacturer (Agilent). Images were scanned immediately using a DNA microarray scanner (Agilent) and processed using Feature Extraction Software 9.5.1 (Agilent) and a standard CGH protocol. Processed signal intensities were further normalized using GC-dependent regression and imported into Microsoft Office Excel 2007 for further analysis. Probes with abnormal hybridization behavior (extremely low [2507 probes] or high [5149 probes] signal intensities in both channels) were excluded. The results from two (CTCF) or three (H3K4 methylation marks) independent ChIP-chip experiments were averaged and converted into UCSC Genome Browser tracks (http://genome.ucsc.edu/) for visualization. To detect differentially methylated regions (DMRs), $\log _{10}$ ratios of individual probes from both comparative genome pool hybridizations were subtracted to obtain hypomethylation scores that were either positive (indicating hypomethylation in Treg cells) or negative (indicating hypomethylation in Tconv cells). Hypomethylation scores of two independent experiments were averaged, and regions were counted as hypomethylated if the average hypomethylation score of three consecutive probes was above a threshold of 0.2333 (at least above the 97 th percentile of all scores). Averaged results from both independent experiments were also converted into UCSC Genome Browser tracks (http://genome.ucsc.edu/); all track files are provided in the Supplemental material. ChIP- and MCIp-chip microarray data have been submitted and are available from the NCBI/GEO repository (accession no. GSE14281). Overlaps of the identified DMRs with conserved sequence elements predicted by the phastCons program based on a whole-genome alignment of vertebrates and with DNase I hypersensitive sites (DHS) in $\mathrm{CD}^{+}$ T-cells (Boyle et al. 2008) were determined using the Table Browser at the UCSC Genome Browser Web site (http://genome.ucsc.edu/). DMRs were classified into three categories according to their CpG content using the promoter definitions proposed by Weber et al. (2007): HCRs (high-CpG regions) contain a CpG ratio $>0.75$ and GC content $>55 \%$ within a 500-bp DMR-centered region; LCRs (low-CpG regions) do not contain a 500-bp area with a CpG ratio >0.48; and ICRs (intermediate $\mathrm{CpG}$ regions) are neither HCRs nor LCRs. Enriched transcription factor consensus motifs in DMRs (500-bp regions) were identified using the RegionMiner tool (http://www.genomatix.de).

\section{Mass spectrometry analysis of bisulfite-converted DNA}

We chose a set of genomic regions based on the MCIp microarray results and designed 95 amplicons for bisulfite conversion. Genomic sequences were extracted from the UCSC genome browser (http://www.genome.ucsc.edu/). PCR primers were designed using the Epidesigner web tool (http://www.epidesigner.com/). For each reverse primer, an additional T7 promoter tag for in vivo transcription was added, as well as a 10-mer tag on the forward primer to adjust for melting temperature differences. All primers were purchased from Sigma-Aldrich (for sequences, see Supplemental Table S4). Sodium bisulfite conversion was performed using an EZ DNA methylation kit (Zymo Research) with $1 \mu \mathrm{g}$ of genomic DNA

\section{Genome Research}

www.genome.org 
and an alternative conversion protocol. Amplification of target regions was followed by SAP treatment, reverse transcription, and subsequent RNA base-specific cleavage (MassCLEAVE, Sequenom) as previously described (Ehrich et al. 2005). Cleavage products were loaded onto silicon chips (spectroCHIP, Sequenom) and analyzed by MALDI-TOF mass spectrometry (MassARRAY Compact MALDI-TOF, Sequenom). Methylation was quantified from mass spectra using the Epityper software v1.0 (Sequenom).

\section{Reporter assays}

DMR regions (800-1200 bp) were PCR-amplified from human genomic DNA and cloned directly into the CpG-free pCpGLCMV/EF1 vector (Klug and Rehli 2006) by ligation or using the InFusion cloning system (Clontech), replacing the CMV enhancer with the DMR regions. Primer sequences are given in Supplemental Table S6. All inserts were verified by sequencing. Luciferase reporter constructs were either mock-treated or methylated in vitro with SssI methylase for $4 \mathrm{~h}$ at $37^{\circ} \mathrm{C}$ and purified with the Plasmid Quick Pure Kit (Macherey-Nagel). One million Jurkat cells were transfected using DEAE-dextran and $1.0 \mu \mathrm{g}$ of each reporter plasmid, and $0.15 \mu \mathrm{g}$ of Renilla control vector. After transfection, cells were left untreated or were stimulated with $20 \mathrm{ng} / \mathrm{mL}$ PMA and $1 \mu \mathrm{M}$ ionomycin or with PHA $(1 \mu \mathrm{g} / \mathrm{mL})$ alone. Triplicate transfections were harvested after $24 \mathrm{~h}$. Cell lysates were assayed for firefly and Renilla luciferase activities using the Dual-Luciferase Reporter Assay System (Promega) on a Lumat LB9501 (Berthold). Firefly luciferase activity of individual transfections was normalized against Renilla luciferase activity.

\section{Acknowledgments}

We thank Ruediger Eder for excellent cell sorting, Lucia Schwarzfischer and Dagmar Glatz for technical assistance, and V. Lobanenkow for providing the anti-CTCF antibodies. This work was supported by grants from the Deutsche Forschungsgemeinschaft to M.E. (KFO146) and to M.R. and P.H. (Re1310/10). The cell sorting facility was funded by the José Carreras Foundation (M.E.).

\section{References}

Antov A, Yang L, Vig M, Baltimore D, Van Parijs L. 2003. Essential role for STAT5 signaling in $\mathrm{CD} 25^{+} \mathrm{CD} 4^{+}$regulatory T cell homeostasis and the maintenance of self-tolerance. J Immunol 171: 3435-3441.

Attanasio C, Reymond A, Humbert R, Lyle R, Kuehn MS, Neph S, Sabo PJ, Goldy J, Weaver M, Haydock A, et al. 2008. Assaying the regulatory potential of mammalian conserved non-coding sequences in human cells. Genome Biol 9: R168. doi: 10.1186/gb-2008-9-12-r168.

Baron U, Floess S, Wieczorek G, Baumann K, Grutzkau A, Dong J, Thiel A, Boeld TJ, Hoffmann P, Edinger M, et al. 2007. DNA demethylation in the human FOXP3 locus discriminates regulatory T cells from activated $\mathrm{FOXP}^{+}{ }^{+}$conventional T cells. Eur J Immunol 37: 2378-2389.

Barski A, Cuddapah S, Cui K, Roh TY, Schones DE, Wang Z, Wei G, Chepelev I, Zhao K. 2007. High-resolution profiling of histone methylations in the human genome. Cell 129: 823-837.

Bell AC, Felsenfeld G. 2000. Methylation of a CTCF-dependent boundary controls imprinted expression of the Igf2 gene. Nature 405: 482-485.

Boyle AP, Davis S, Shulha HP, Meltzer P, Margulies EH, Weng Z, Furey TS, Crawford GE. 2008. High-resolution mapping and characterization of open chromatin across the genome. Cell 132: 311-322.

Brandeis M, Frank D, Keshet I, Siegfried Z, Mendelsohn M, Nemes A, Temper V, Razin A, Cedar H. 1994. Sp1 elements protect a CpG island from de novo methylation. Nature 371: 435-438.

Chong MM, Rasmussen JP, Rudensky AY, Littman DR. 2008. The RNAseIII enzyme Drosha is critical in T cells for preventing lethal inflammatory disease. J Exp Med 205: 2005-2017.

Dovat S, Montecino-Rodriguez E, Schuman V, Teitell MA, Dorshkind K, Smale ST. 2005. Transgenic expression of helios in B lineage cells alters B cell properties and promotes lymphomagenesis. J Immunol 175: 35083515 .
Ehrich M, Nelson MR, Stanssens P, Zabeau M, Liloglou T, Xinarianos G, Cantor CR, Field JK, and van den Boom D. 2005. Quantitative highthroughput analysis of DNA methylation patterns by base-specific cleavage and mass spectrometry. Proc Natl Acad Sci 102: 15785-15790.

Floess S, Freyer J, Siewert C, Baron U, Olek S, Polansky J, Schlawe K, Chang HD, Bopp T, Schmitt E, et al. 2007. Epigenetic control of the foxp3 locus in regulatory T cells. PLoS Biol 5: e38. doi: 10.1371/ journal.pbio.0050038.

Gebhard C, Schwarzfischer L, Pham TH, Schilling E, Klug M, Andreesen R, Rehli M. 2006. Genome-wide profiling of CpG methylation identifies novel targets of aberrant hypermethylation in myeloid leukemia. Cancer Res 66: 6118-6128.

Goto T, Monk M. 1998. Regulation of X-chromosome inactivation in development in mice and humans. Microbiol Mol Biol Rev 62: 362-378.

Heintzman ND, Stuart RK, Hon G, Fu Y, Ching CW, Hawkins RD, Barrera LO, Van Calcar S, Qu C, Ching KA, et al. 2007. Distinct and predictive chromatin signatures of transcriptional promoters and enhancers in the human genome. Nat Genet 39: 311-318.

Herman JG, Baylin SB. 2003. Gene silencing in cancer in association with promoter hypermethylation. N Engl J Med 349: 2042-2054.

Hill JA, Feuerer M, Tash K, Haxhinasto S, Perez J, Melamed R, Mathis D, Benoist C. 2007. Foxp3 transcription-factor-dependent and independent regulation of the regulatory $\mathrm{T}$ cell transcriptional signature. Immunity 27: 786-800.

Hoffmann P, Eder R, Kunz-Schughart LA, Andreesen R, Edinger M. 2004 Large-scale in vitro expansion of polyclonal human CD $4{ }^{+} \mathrm{CD} 25^{\text {high }}$ regulatory T cells. Blood 104: 895-903.

Hoffmann P, Eder R, Boeld TJ, Doser K, Piseshka B, Andreesen R, Edinger M. 2006. Only the CD45RA ${ }^{+}$subpopulation of $\mathrm{CD} 4^{+} \mathrm{CD} 25^{\text {high }} \mathrm{T}$ cells gives rise to homogeneous regulatory T-cell lines upon in vitro expansion. Blood 108: 4260-4267.

Hoogenkamp M, Krysinska H, Ingram R, Huang G, Barlow R, Clarke D, Ebralidze A, Zhang P, Tagoh H, Cockerill PN, et al. 2007. The Pu.1 locus is differentially regulated at the level of chromatin structure and noncoding transcription by alternate mechanisms at distinct developmental stages of hematopoiesis. Mol Cell Biol 27: 7425-7438.

Hou J, Schindler U, Henzel WJ, Wong SC, McKnight SL. 1995. Identification and purification of human Stat proteins activated in response to interleukin-2. Immunity 2: 321-329.

Hsueh YP, Liang HE, Ng SY, Lai MZ. 1997. CD28-costimulation activates cyclic AMP-responsive element-binding protein in T lymphocytes. J Immunol 158: 85-93.

Illingworth R, Kerr A, Desousa D, Jorgensen H, Ellis P, Stalker J, Jackson D, Clee C, Plumb R, Rogers J, et al. 2008. A novel CpG island set identifies tissue-specific methylation at developmental gene loci. PLoS Biol 6: e22. doi: 10.1371/journal.pbio.0060022.

Kim HP, Leonard WJ. 2007. CREB/ATF-dependent T cell receptor-induced FoxP3 gene expression: A role for DNA methylation. J Exp Med 204: 1543-1551.

Klug M, Rehli M. 2006. Functional analysis of promoter CpG methylation using a CpG-free luciferase reporter vector. Epigenetics 1: 127-130.

Li E, Beard C, Jaenisch R. 1993. Role for DNA methylation in genomic imprinting. Nature 366: 362-365.

Liston A, Lu LF, O'Carroll D, Tarakhovsky A, Rudensky AY. 2008. Dicerdependent microRNA pathway safeguards regulatory T cell function. $J$ Exp Med 205: 1993-2004.

Meissner A, Mikkelsen TS, Gu H, Wernig M, Hanna J, Sivachenko A, Zhang X, Bernstein BE, Nusbaum C, Jaffe DB, et al. 2008. Genome-scale DNA methylation maps of pluripotent and differentiated cells. Nature 454: 766-770.

Metivier R, Penot G, Hubner MR, Reid G, Brand H, Kos M, Gannon F. 2003. Estrogen receptor-alpha directs ordered, cyclical, and combinatorial recruitment of cofactors on a natural target promoter. Cell 115: 751-763.

Mohn F, Weber M, Rebhan M, Roloff TC, Richter J, Stadler MB, Bibel M, and Schubeler D. 2008. Lineage-specific polycomb targets and de novo DNA methylation define restriction and potential of neuronal progenitors. Mol Cell 30: 755-766.

Okano M, Bell DW, Haber DA, Li E. 1999. DNA methyltransferases Dnmt3a and Dnmt3b are essential for de novo methylation and mammalian development. Cell 99: 247-257.

Pfoertner S, Jeron A, Probst-Kepper M, Guzman CA, Hansen W, Westendorf AM, Toepfer T, Schrader AJ, Franzke A, Buer J, et al. 2006. Signatures of human regulatory T cells: An encounter with old friends and new players. Genome Biol 7: R54. doi: 10.1186/gb-2006-7-7-r54.

Plass C, Soloway PD. 2002. DNA methylation, imprinting and cancer. Eur J Hum Genet 10: 6-16.

Polansky JK, Kretschmer K, Freyer J, Floess S, Garbe A, Baron U, Olek S, Hamann A, von Boehmer H, Huehn J. 2008. DNA methylation controls Foxp3 gene expression. Eur J Immunol 38: 1654-1663.

Schilling E, Rehli M. 2007. Global, comparative analysis of tissue-specific promoter CpG methylation. Genomics 90: 314-323. 


\section{Schmidl et al.}

Schoenborn JR, Dorschner M, Sekimata M, Santer D, Shnyreva M, Fitzpatrick DR, Stamatoyannopoulos JA, Wilson CB. 2007. Comprehensive epigenetic profiling identifies multiple distal regulatory elements directing Ifng transcription. Nat Immunol 8: 732-742.

Song F, Mahmood S, Ghosh S, Liang P, Smiraglia DJ, Nagase H, Held WA. 2008. Tissue specific differentially methylated regions (TDMR): Changes in DNA methylation during development. Genomics 93: 130-139.

van Kooten C, Banchereau J. 1997. Functions of CD40 on B cells, dendritic cells and other cells. Curr Opin Immunol 9: 330-337.

Vignali DA, Collison LW, Workman CJ. 2008. How regulatory T cells work. Nat Rev Immunol 8: 523-532.

Walsh CP, Chaillet JR, Bestor TH. 1998. Transcription of IAP endogenous retroviruses is constrained by cytosine methylation. Nat Genet 20: 116-117.

Wang R, Wan Q, Kozhaya L, Fujii H, Unutmaz D. 2008a. Identification of a regulatory $\mathrm{T}$ cell specific cell surface molecule that mediates suppressive signals and induces Foxp3 expression. PLoS One 3: e2705. doi: 10.1371/journal.pone.0002705.

Wang Z, Zang C, Rosenfeld JA, Schones DE, Barski A, Cuddapah S, Cui K, Roh TY, Peng W, Zhang MQ, et al. 2008b. Combinatorial patterns of histone acetylations and methylations in the human genome. Nat Genet 40: 897-903.

Weber M, Hellmann I, Stadler MB, Ramos L, Paabo S, Rebhan M, Schubeler D. 2007. Distribution, silencing potential and evolutionary impact of promoter DNA methylation in the human genome. Nat Genet 39: 457466 .

Wing K, Onishi Y, Prieto-Martin P, Yamaguchi T, Miyara M, Fehervari Z, Nomura T, Sakaguchi S. 2008. CTLA-4 control over Foxp3 ${ }^{+}$regulatory T cell function. Science 322: 271-275.

Zhou X, Jeker LT, Fife BT, Zhu S, Anderson MS, McManus MT, Bluestone JA 2008. Selective miRNA disruption in T reg cells leads to uncontrolled autoimmunity. J Exp Med 205: 1983-1991.

Zorn E, Nelson EA, Mohseni M, Porcheray F, Kim H, Litsa D, Bellucci R, Raderschall E, Canning C, Soiffer RJ, et al. 2006. IL-2 regulates FOXP3 expression in human $\mathrm{CD} 4{ }^{+} \mathrm{CD} 25^{+}$regulatory $\mathrm{T}$ cells through a STATdependent mechanism and induces the expansion of these cells in vivo. Blood 108: 1571-1579.

Received January 21, 2009; accepted in revised form April 20, 2009.

1174 Genome Research www.genome.org 


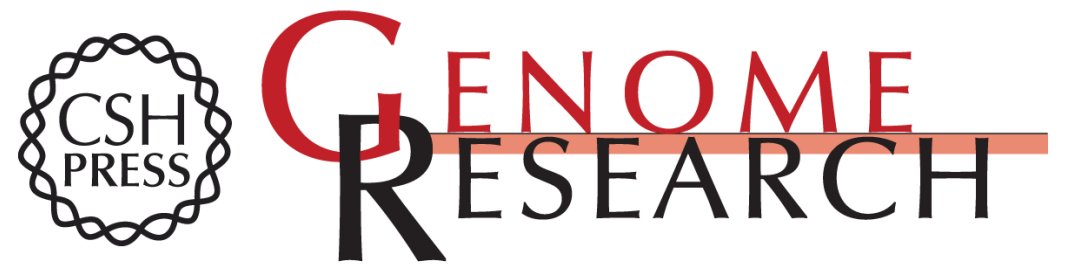

\section{Lineage-specific DNA methylation in T cells correlates with histone methylation and enhancer activity}

Christian Schmidl, Maja Klug, Tina J. Boeld, et al.

Genome Res. 2009 19: 1165-1174 originally published online June 3, 2009

Access the most recent version at doi:10.1101/gr.091470.109

Supplemental Material

References

License

Email Alerting Service
http://genome.cshlp.org/content/suppl/2009/06/08/gr.091470.109.DC1

This article cites 45 articles, 15 of which can be accessed free at: http://genome.cshlp.org/content/19/7/1165.full.html\#ref-list-1

Receive free email alerts when new articles cite this article - sign up in the box at the top right corner of the article or click here.

\section{Affordable, Accurate Sequencing.}

